\title{
Noise Model Analysis and Estimation of Effect due to Wind Driven Ambient Noise in Shallow Water
}

\author{
S. Sakthivel Murugan, ${ }^{1}$ V. Natarajan, ${ }^{2}$ and R. Rajesh Kumar ${ }^{1}$ \\ ${ }^{1}$ Department of ECE, SSN College of Engineering, Tamilnadu, Chennai 603110, India \\ ${ }^{2}$ Department of Instrumentation Engineering, MIT Campus, Anna University, Chennai 600044, India
}

Correspondence should be addressed to S. Sakthivel Murugan, sakthivels@ssn.edu.in

Received 1 June 2011; Revised 27 September 2011; Accepted 28 September 2011

Academic Editor: Robert Frouin

Copyright (C) 2011 S. Sakthivel Murugan et al. This is an open access article distributed under the Creative Commons Attribution License, which permits unrestricted use, distribution, and reproduction in any medium, provided the original work is properly cited.

Signal transmission in ocean using water as a channel is a challenging process due to attenuation, spreading, reverberation, absorption, and so forth, apart from the contribution of acoustic signals due to ambient noises. Ambient noises in sea are of two types: manmade (shipping, aircraft over the sea, motor on boat, etc.) and natural (rain, wind, seismic, etc.), apart from marine mammals and phytoplanktons. Since wind exists in all places and at all time: its effect plays a major role. Hence, in this paper, we concentrate on estimating the effects of wind. Seven sets of data with various wind speeds ranging from $2.11 \mathrm{~m} / \mathrm{s}$ to $6.57 \mathrm{~m} / \mathrm{s}$ were used. The analysis is performed for frequencies ranging from $100 \mathrm{~Hz}$ to $8 \mathrm{kHz}$. It is found that a linear relationship between noise spectrum and wind speed exists for the entire frequency range. Further, we developed a noise model for analyzing the noise level. The results of the empirical data are found to fit with results obtained with the aid of noise model.

\section{Introduction}

Ocean ambient noise is an inherent characteristic of the medium having no specific point source. It is the residual noise background in the absence of individual identifiable sources that may be considered as the natural noise environment for hydrophone sensors. It comprises of number of components that contribute to the noise level (NL) in varying degrees depending on the location of measurements [1]. The sources include geological disturbances, nonlinear wave interaction, turbulent wind stress on the sea surface, shipping, distant storms, seismic prospecting, marine animals, breaking waves, spray, rain, hail impacts, and turbulence [2]. In general, over a broad frequency range, the ambient noise spectrum characteristic varies depending on the sources and conditions prevailing at the measurement location.

A direct connection between wind force and the level of ambient noise is observed for a frequency range of $500 \mathrm{~Hz}$ to $25 \mathrm{kHz}$. Noise level spectrum is summarized in [3]. Knudsen spectra [4], show the strong dependence of spectral power level with wind speed and sea states.
The work on spectra and sources of ambient noise in the ocean observed a decrease in wind/sea state dependency of underwater ambient noise below $500 \mathrm{~Hz}$. Further, it was shown that there was no dependency below $100 \mathrm{~Hz}$, and the wind-generated noise measurement shows self-similar spectra between $100 \mathrm{~Hz}$ to $10 \mathrm{kHz}[3,4]$.

It is inferred that the effect of wind speed is dominant in the frequency band of $22 \mathrm{~Hz}$ to $5 \mathrm{kHz}$ by experimenting at 40 different locations [5]. Further high frequency noise is said to be highly correlated to wind speed from $4-15 \mathrm{~m} / \mathrm{sec}$. The optimal frequency range for estimating wind is from $2-6 \mathrm{kHz}$ [6].

Ambient noise is the sustained unwanted background noise prevailing at any location. This masks the signals from underwater acoustic instruments. So the detection of background noise is essential to enhance the signal-to-Noise ratio (SNR) of acoustic-based underwater instruments. Hence, measurement and characterization of ambient noise forms a significant part in any underwater activity. The interest in ambient noise characterization has grown and presently is of much interest to engineers working in the fields of active and passive sonars, underwater sensors, and signal processing. 


\section{Data Collection and Algorithm}

2.1. Data Collection. Noise measurements were made using two calibrated omnidirectional hydrophones mounted in a vertical array at $5 \mathrm{~m}$ and $15 \mathrm{~m}$ depths. The hydrophones were suspended from the measurement platform using the rope and mounting arrangement that links to the rope. The hydrophones have a receiving sensitivity of $-170 \mathrm{~dB}$ over a frequency range between $0.1 \mathrm{~Hz}$ and $120 \mathrm{kHz}$. The data were acquired at a rate of $50 \mathrm{kHz}$ and $500 \mathrm{kHz}$, filtered and digitized with portable data acquisition system with 12-bit resolution. During the period of data collection, all machinery on the boat/ship was switched off and the recording system was powered by battery. The wind speed was simultaneously measured during each sampling. The measurement consists of 7 sets of data. The wind speeds of collected data range from $2.11 \mathrm{~m} / \mathrm{s}$ to $6.57 \mathrm{~m} / \mathrm{s}$. Measurements showing the evidence of noise from non-wind-dependent sources such as rain, dolphin, and ship were not included in the analysis.

2.2. Algorithm. Theoretically the relationship between the noise levels is assumed to be linear to the logarithm of the wind speed [7], and this can be expressed as

$$
\mathrm{NL}=B+20 n \log (U)
$$

where NL and $U$ stand for noise level and wind speed, respectively. The constants $B$ and $n$ were determined by fitting the experimental data to the model at different frequencies. $n$ is obtained from $1 / 20$ th slope of regression line and the ordinate intercept of the line gives $B$ for each empirical fit. The spectral analysis was carried out in MATLAB using Welch method of averaging periodogram. The frequency range of interest for the current study was from $500 \mathrm{~Hz}$ to $8 \mathrm{kHz}$ where the best correlation between the wind speed and the noise level has been observed.

\section{Results and Discussion}

Analysis has been carried out to study wind-dependent ambient noise spectrum level in the frequency range between $500 \mathrm{~Hz}$ and $8 \mathrm{kHz}$. Noise spectrum for seven different wind speeds $[2.11,3.32,5.92,6.03,6.06,6.16$, and $6.57 \mathrm{~m} / \mathrm{s}]$ is shown in Figure 1. It is observed that the noise level increases with increase in wind speed.

Figure 2 is also known as regression plot. It is observed from Figure 2 that there is a steep increase in the slope and noise level as wind speed increases. Table 1 shows the values of $B$ and $n$ obtained from regression plots. The value of slope is maximum at $500 \mathrm{~Hz}$ and decreases as frequency increases. The value of $n$ and $B$ obtained from the empirical fitting is used for validation with measured noise level.

Figures 3, 4, 5, and 6 show the comparison of predicted and measured noise levels for wind speed of $2.11 \mathrm{~m} / \mathrm{s}$, $3.32 \mathrm{~m} / \mathrm{s}, 5.92 \mathrm{~m} / \mathrm{s}$, and $6.57 \mathrm{~m} / \mathrm{s}$, respectively. It is seen that the predicted noise level is in good agreement with the measured noise levels. As the wind speed increases, the predicted noise model deviates from the measured noise level.

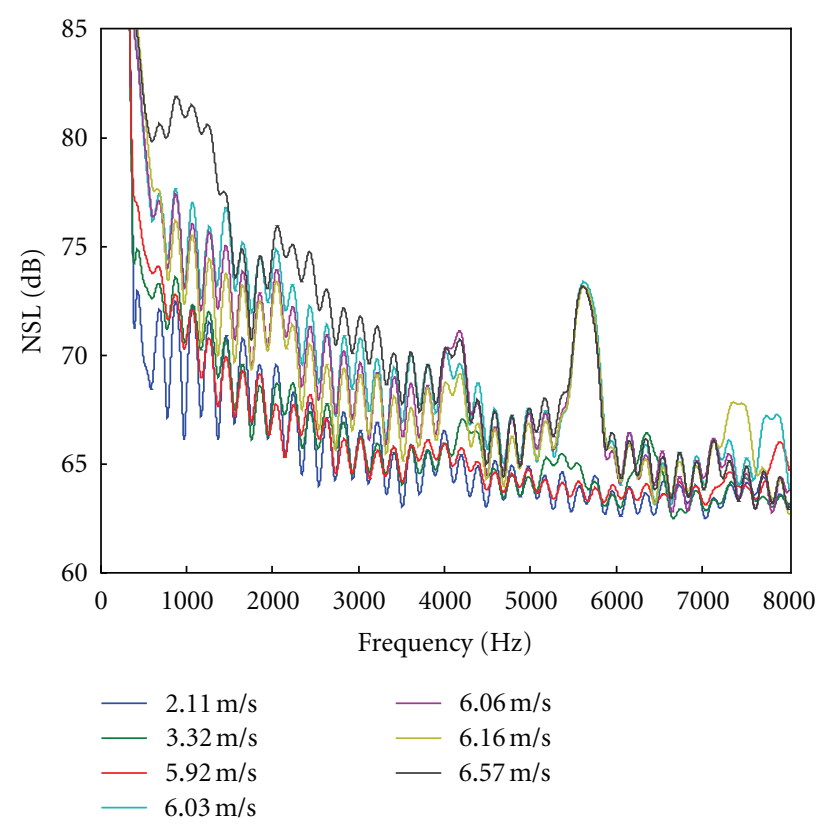

FIGURE 1: Noise spectrum at different wind speeds.

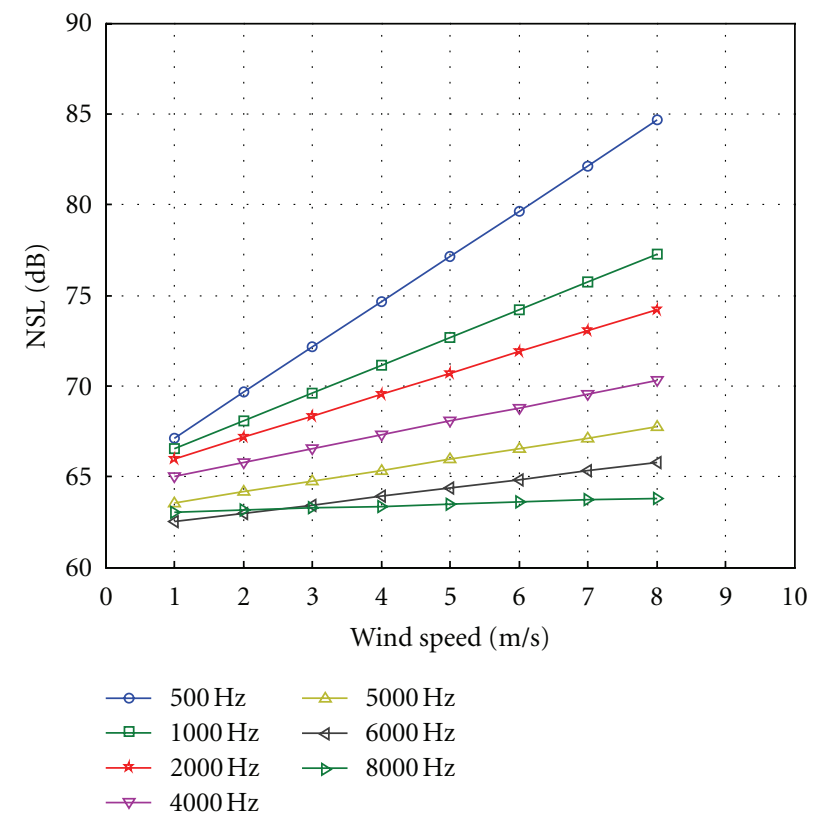

Figure 2: Noise level at different frequencies for varying wind speeds.

\section{Conclusion}

In this paper, an estimation of power spectral density of ambient noise due to wind at various speeds ranging from $2.11 \mathrm{~m} / \mathrm{s}$ to $6.59 \mathrm{~m} / \mathrm{s}$ is analysed and observed that the effect of wind is dominating at lower frequencies from $100 \mathrm{~Hz}$ to $5 \mathrm{KHz}$. A noise model for estimation of effect of wind at different wind speeds for various frequencies was developed and found that it best suits with the practical data. The analysis shows that noise level increases as wind speed increases. 
TABLe 1: Values of $B$ and $n$ from regression plots.

\begin{tabular}{lccccc}
\hline Frequency $(\mathrm{Hz})$ & $B$ & $n$ & Frequency $(\mathrm{Hz})$ & $B$ & $n$ \\
\hline 500 & 67.14 & 0.13 & 4500 & 63.19 & 0.01 \\
1000 & 66.57 & 0.08 & 5000 & 63.56 & 0.03 \\
1500 & 68.16 & 0.05 & 5500 & 61.04 & 0.08 \\
2000 & 65.98 & 0.06 & 6000 & 62.5 & 0.02 \\
2500 & 64.28 & 0.05 & 6500 & 64.06 & 0.003 \\
3000 & 66.93 & 0.02 & 7000 & 62.56 & 0.01 \\
3500 & 62.29 & 0.04 & 7500 & 63.41 & 0.02 \\
4000 & 65.04 & 0.04 & 8000 & 63.04 & 0.006 \\
\hline
\end{tabular}

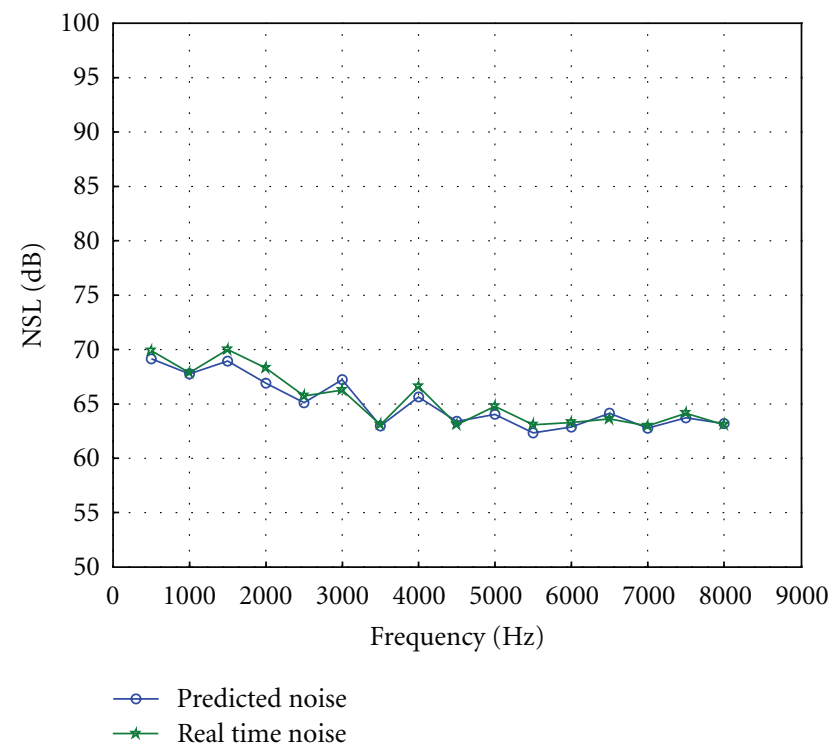

Figure 3: Comparison of predicted and real time noise for wind speed of $2.11 \mathrm{~m} / \mathrm{s}$.

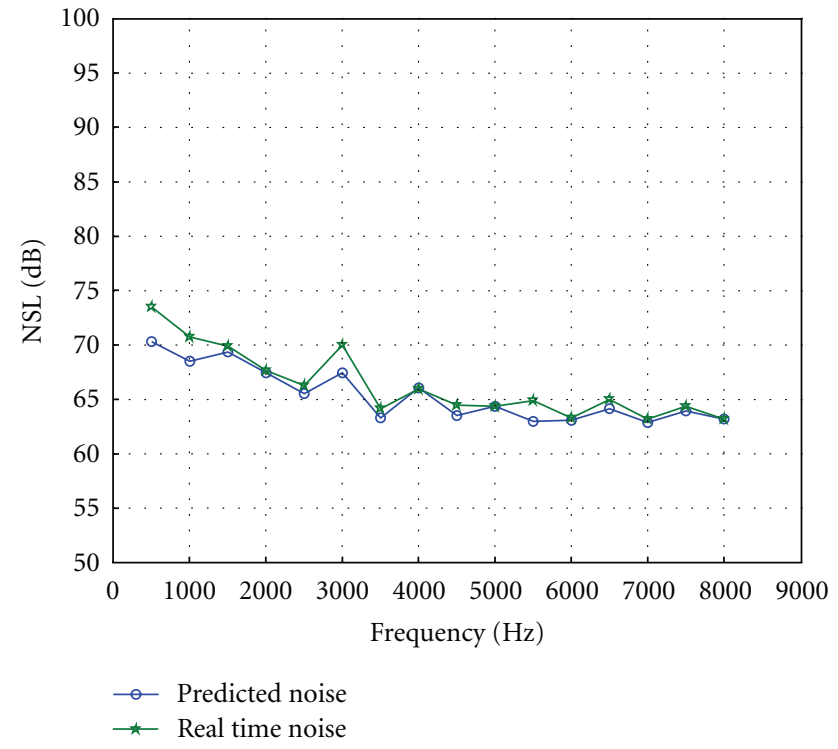

Figure 4: Comparison of predicted and real time noise for wind speed of $3.32 \mathrm{~m} / \mathrm{s}$.

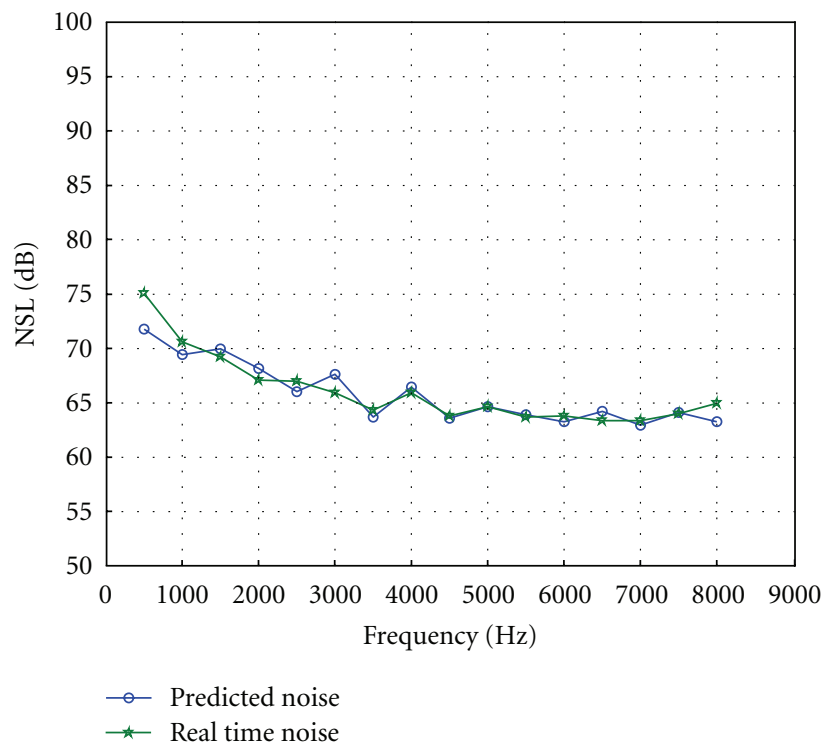

Figure 5: Comparison of predicted and real time noise for wind speed of $5.92 \mathrm{~m} / \mathrm{s}$.

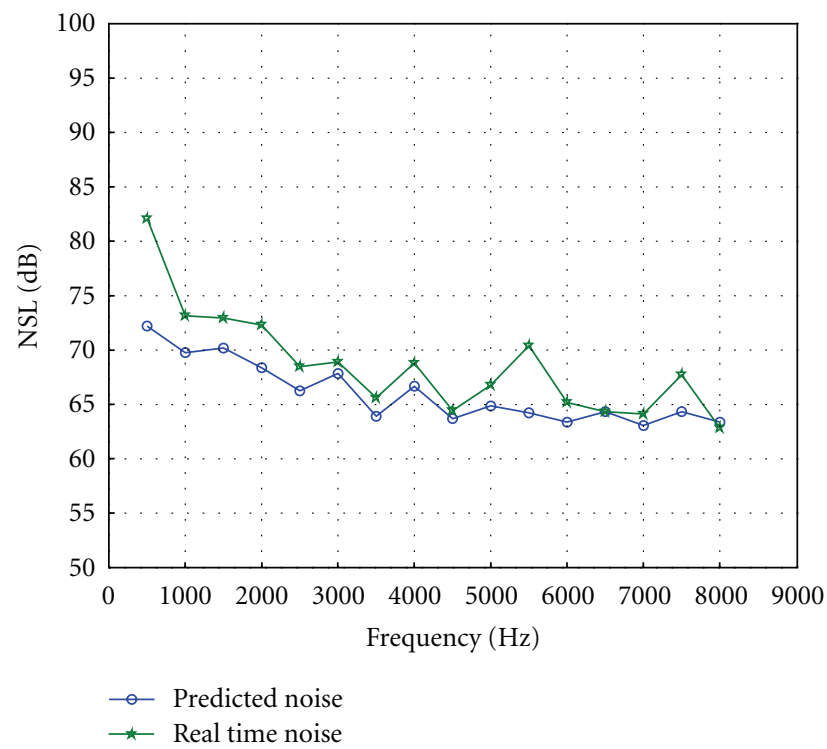

FIGURE 6: Comparison of predicted and real time noise for wind speed of $6.57 \mathrm{~m} / \mathrm{s}$. 
There was good correlation between wind speed and noise level in the frequency range between $500 \mathrm{~Hz}$ to $8000 \mathrm{~Hz}$.

\section{Acknowledgments}

The data for the above analysis is provided by National Institute of Ocean Technology, Chennai. The authors wish to acknowledge the valuable support given by Dr. G. Latha, Project Director, Ocean Observation and Acoustic System, NIOT for the excellent contribution of the relevant data for processing. The authors also wish to thank Dr. S. Radha HOD, ECE Department for her support.

\section{References}

[1] R. J. Urick, Principles of Underwater Sound, McGraw-Hill, New York, NY, USA, 3rd edition, 1983.

[2] R. A. Wagstaff, "An ambient noise model for the northeast pacific ocean basin," IEEE Journal of Oceanic Engineering, vol. 30, no. 2, pp. 286-294, 2005.

[3] G. M. Wenz, "Acoustic ambient noise in the ocean: spectra and sources," Journal of Acoustic Society of America, vol. 30, pp. 1936-1956, 1962.

[4] V. O. Knudsen, R. S. Alford, and J. W Emling, "Underwater ambient noise," Journal of Marine Research, vol. 7, pp. 410-429, 1948.

[5] D. H. Cato, "Ambient sea noise in water near Australia," Journal of the Acoustical Society of America, vol. 60, no. 2, pp. 320-328, 1976.

[6] L. Zedel, L. Gordon, and S. Osterhus, "Ocean Ambient Sound Instrument System: acoustic estimation of wind speed and direction from a subsurface package," Journal of Atmospheric and Oceanic Technology, vol. 16, no. 8, pp. 1118-1126, 1999.

[7] C. L. Piggott, "Ambient Sea noise at low frequencies in shallow water of the Scotian Shelf," Journal of Acoustic Society of America, vol. 36, pp. 2152-2163, 1964. 

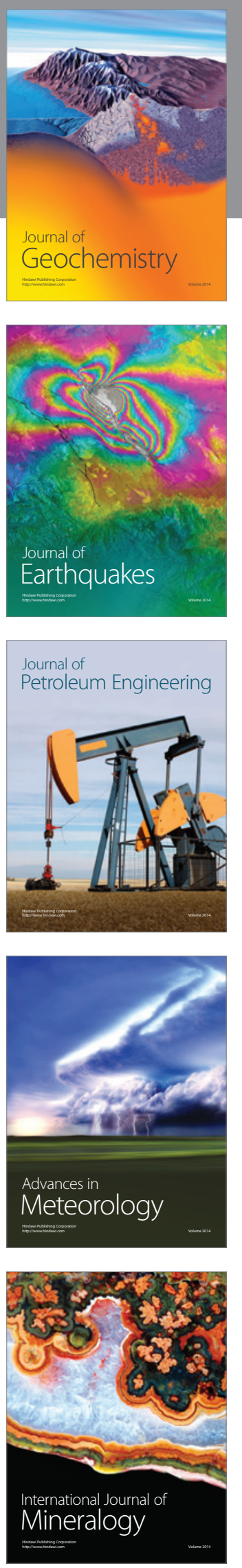
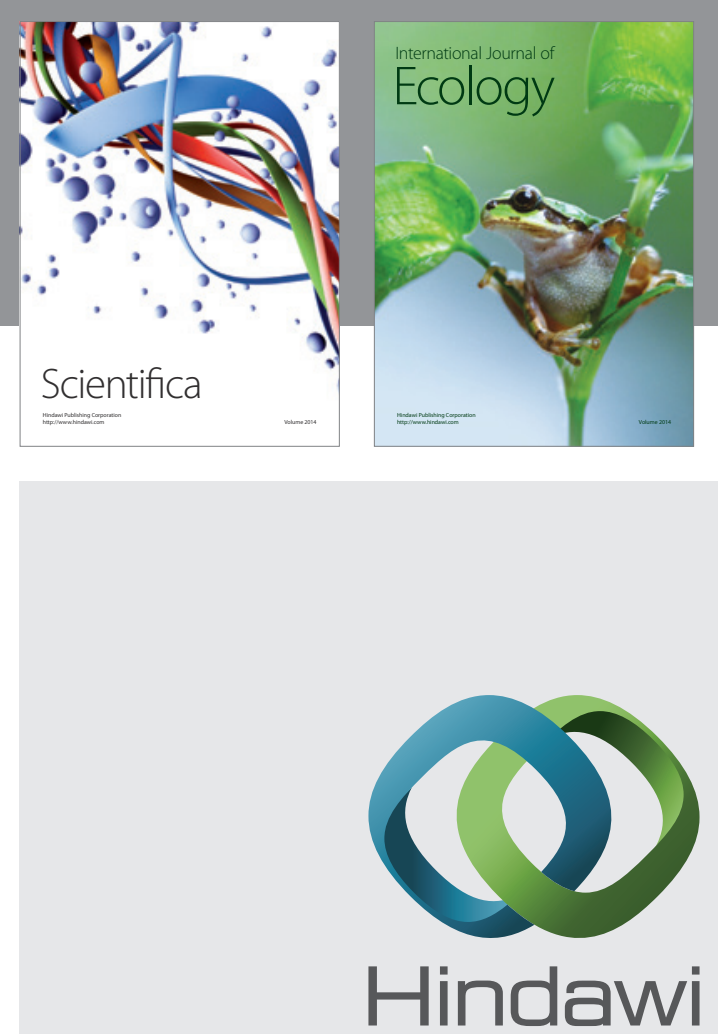

Submit your manuscripts at http://www.hindawi.com
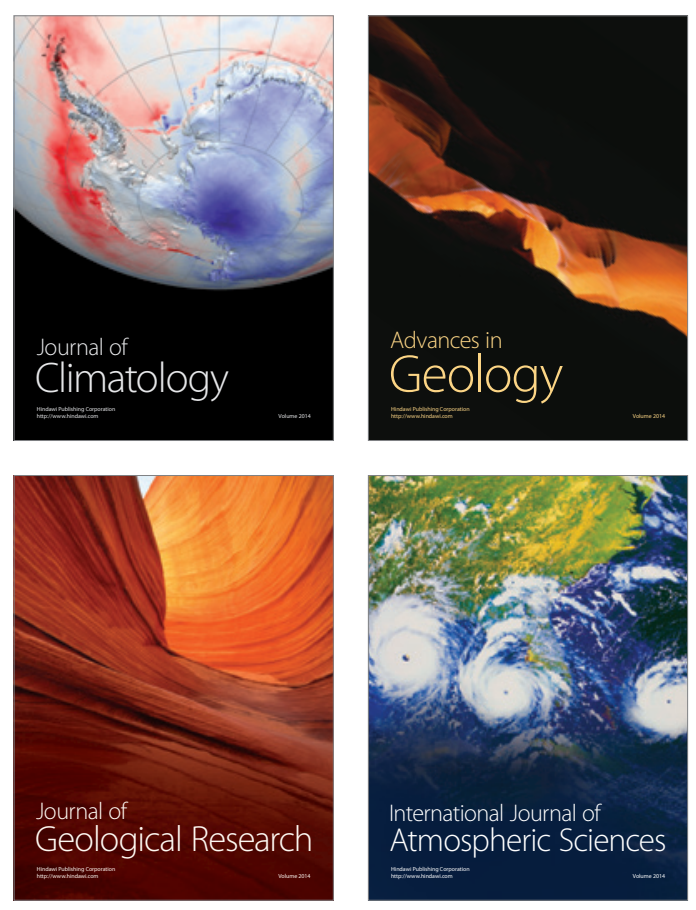
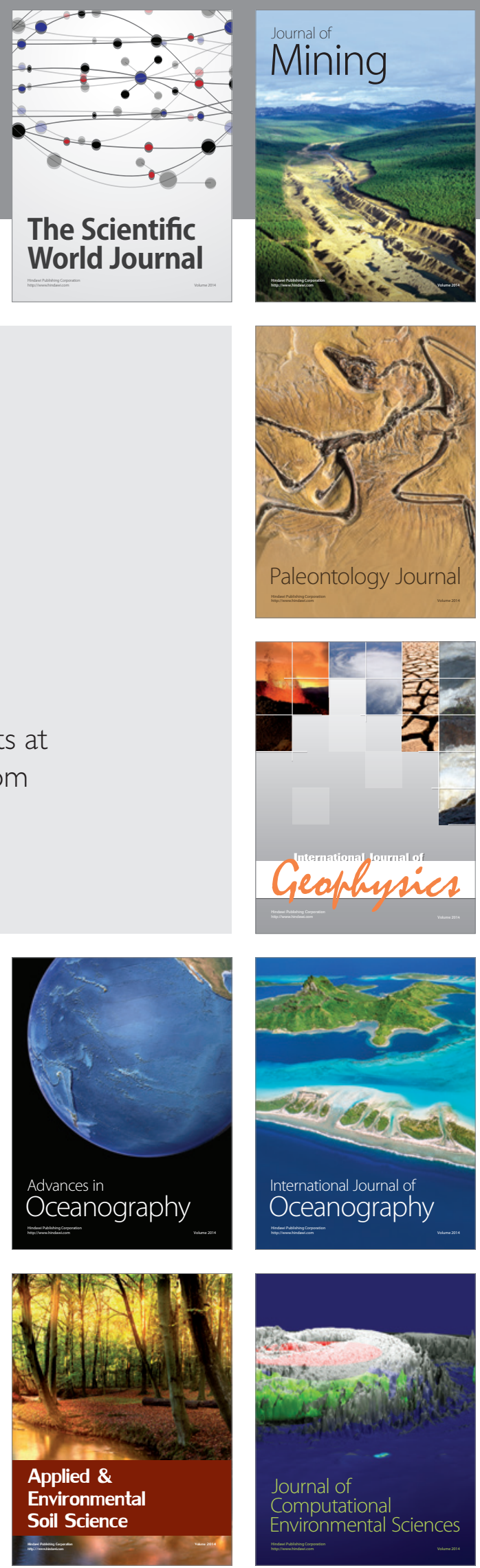